\title{
FAMILIAL CARDIOMEGALY
}

BY

\author{
R. T. GAUNT AND M. A. LECUTIER \\ From the Departments of Pathology and Medicine, Royal Hospital, Chesterfield \\ Received July 9, 1955
}

William Evans (1949) described a series of cases of cardiac enlargement in which none of the usual causes of cardiomegaly was present, and among this series of nine cases there were three who were related (a mother and two sons). Moreover, the mode of death in several relatives suggested that a similar condition may have been present in other members of the family. A similar family history had been reported previously in patients with unexplained cardiomegaly on two occasions (Case Records, Massachusetts General Hospital, 1942) and Addarii and Mahaim (1946). A necropsy carried out on one member of the family described by Evans showed an unusual finding in that there were extensive glycogen deposits in the heart muscle; in addition there were areas of dense fibrosis with hypertrophy of remaining fibres. Similar deposits of glycogen were also found in the vastus externus muscle. Glycogen is found in the heart muscle in some cases of Von-Gierke's disease but Evans did not consider his case to be an example of this condition as there was no evidence of a failure of glycogenolysis. Moreover, death has always occurred at an early age in the recorded cases of Von-Gierke's disease that have shown cardiac involvement. Evans concluded that this was a definite syndrome possibly related to Friedreich's ataxia and suggested that it be called familial cardiomegaly.

The purpose of this paper is to present a further series of cases in which there is a familial incidence and in which there are unusual features similar to those described by Evans. One of the cases has been followed to necropsy.

\section{CASE Histories}

Case 1. A man, aged 49 years, was first seen in August, 1951, when he complained of increasing dyspnœa for the previous three months. Prior to this he had been quite well and had worked in the mines all his life. A month after first noticing shortness of breath, he had two attacks in which he had a transient feeling of faintness and unsteadiness. He ceased working at this time and since then had one further attack. There was no past history of rheumatism.

On clinical examination he had gross cardiac enlargement and a bradycardia (pulse rate 32 a minute). There was no evidence of valvular disease and the blood pressure was 162/90. X-ray examination confirmed the presence of gross cardiac enlargement and a cardiogram (Fig. 1) showed auricular flutter, A-V dissociation, and great increase of amplitude of all complexes. Wasserman and Kahn reactions were negative.

This patient was kept under observation and it was found impossible to affect the rate of his heart by any of the usual measures adopted in heart block. He subsequently developed true StokesAdams attacks and although ephedrine in fairly large doses (4 grains daily) seemed to reduce their frequency there was little change in the cardiographic findings.

Case 2. A woman, aged 23 years, came to the Out-Patient Department in November, 1951, complaining of "rheumatism" for three months. Her rheumatism had started in the interphalangeal joints but had subsequently spread to the shoulders and knees and the pains tended to move from joint to joint; she gave a history of occasional sore throats, and slight shortness of breath 


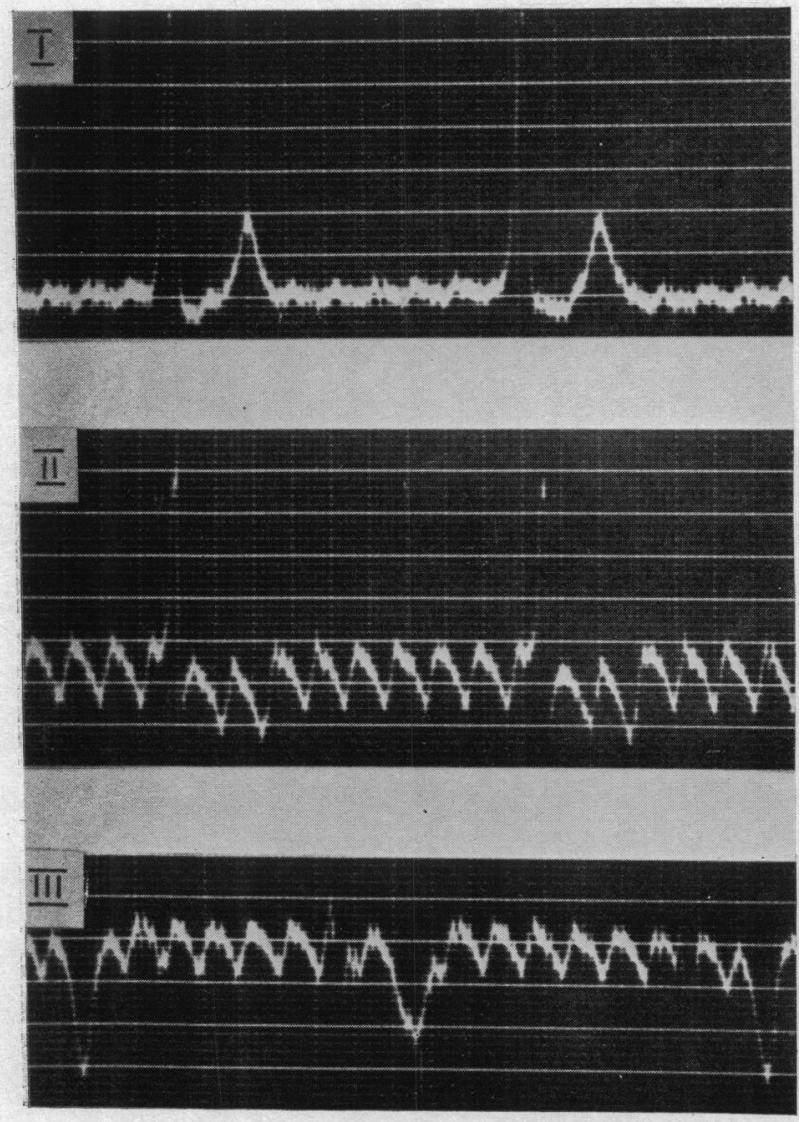

FIG. 1.-Electrocardiogram from Case 1, showing auricular flutter, A-V dissociation, and greatly increased amplitude of all complexes.

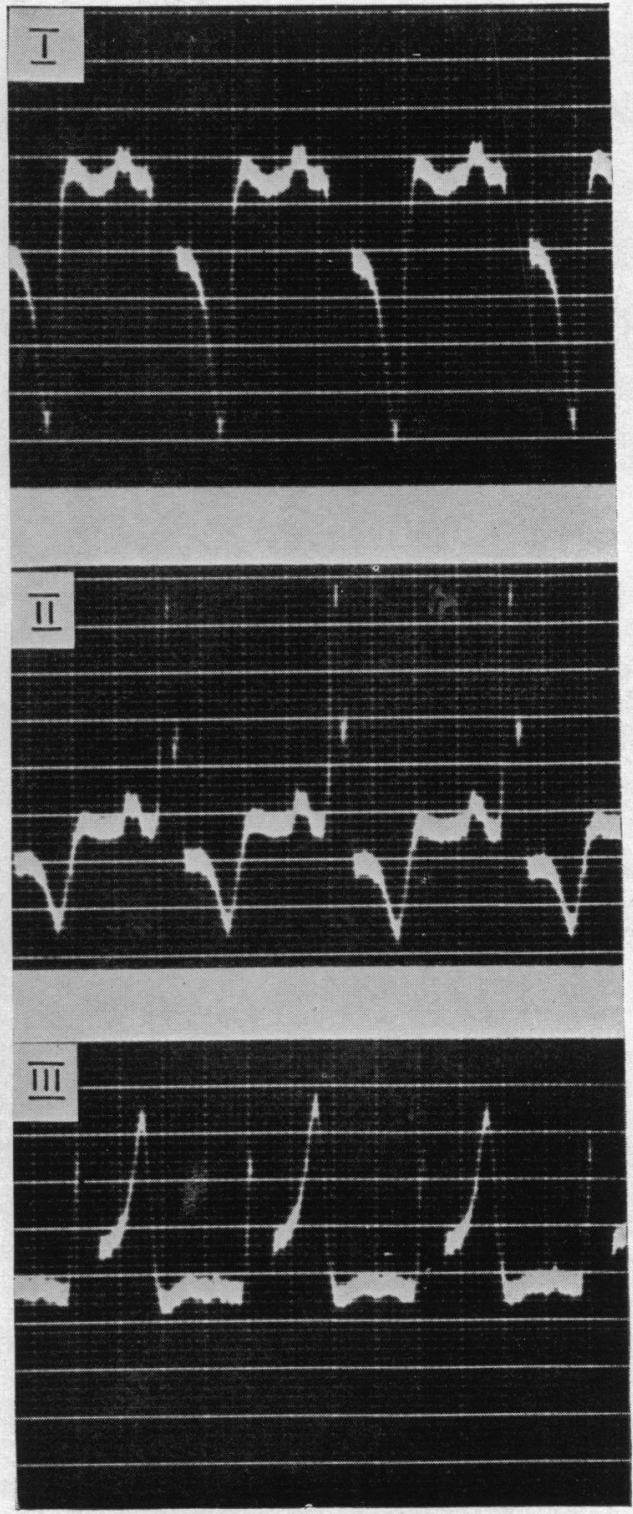

FIG. 2.--Electrocardiogram from Case 2, showing greatly increased amplitude of QRS deflections and $T$ waves, and changes in the $S-T$ segments.

on exertion, but there was no previous history of rheumatism, and she had considered herself quite fit until three months before attending the hospital. On examination there were no obvious joint changes but she was found to have considerable cardiac enlargement and there was also a soft systolic murmur audible both in the mitral and in the pulmonary areas. This was not thought to be of organic significance. Her blood pressure was 138/80. The sedimentation rate was found to be $3 \mathrm{~mm}$. in 1 hour; $7 \mathrm{~mm}$. in 2 hours (Westergren's method). X-ray examination of the chest confirmed the presence of gross cardiomegaly, and the cardiogram (Fig. 2) showed greatly 
increased amplitude of the complexes and also gross abnormality of the S-T segments and T waves. A diagnosis of "isolated myocarditis" was made.

Case 3. A woman, aged 53 years, was seen at her home in April, 1952, when she was having frequent and severe Stokes-Adams attacks which had been present for the previous thirty-six hours. There was a history of "collapse" three years previously and she had then been told by her doctor that her heart was affected. Since then she had been short of breath on exertion. She gave a history of chorea at eight or nine years of age. On examination the pulse rate was 6 a minute and the heart was found to be considerably enlarged. The blood pressure was $150 / 35$. She was admitted to hospital and during the course of the next six weeks the pulse gradually returned to a normal rate and the blood pressure rose to 200/100. An electrocardiogram showed complete heart block with complexes of high potential, and the record during recovery is shown in Fig. 3. Wassermann and Kahn reactions were negative.

When Case 3 was first seen at her home, among the relatives present was her daughter who

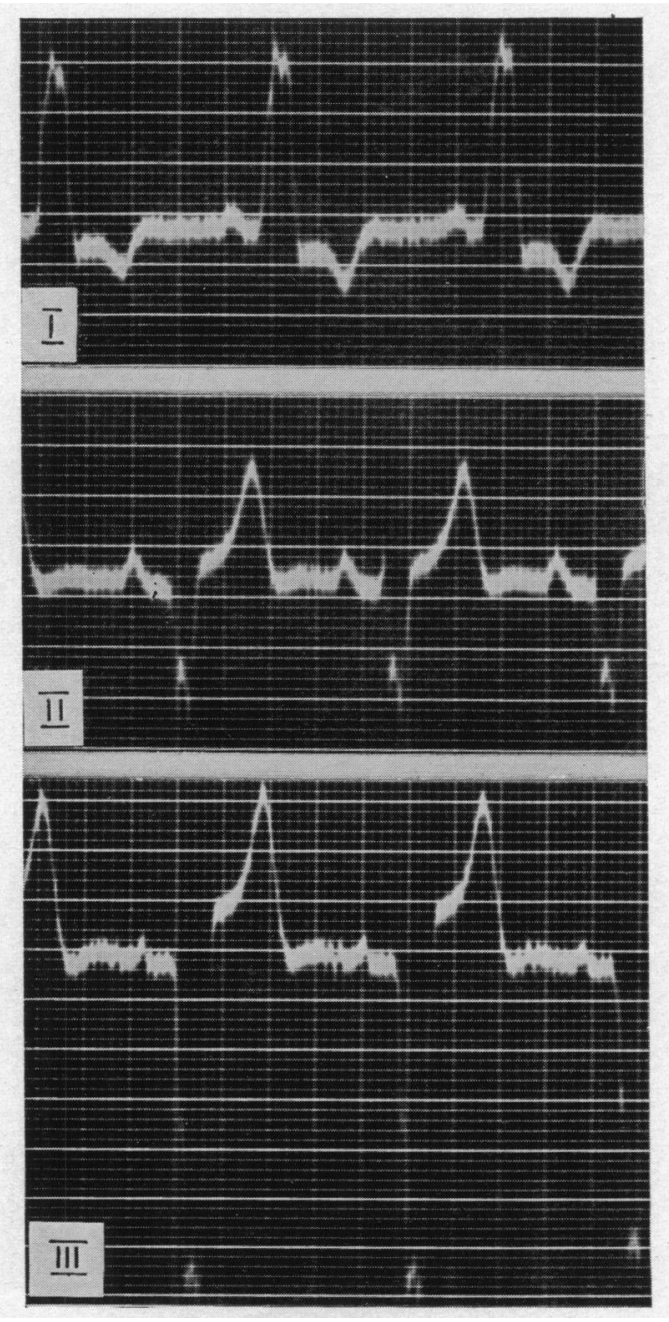

Fig. 3.-Electrocardiogram from Case 3, showing some increase in amplitude of QRS deflections and the $T$ waves of leads II and III and changes in the $S-T$ segments. 
was recognized as being the patient described above as Case 2. It was then learnt that Case 1 was also related being the brother of Case 3 (and therefore maternal uncle of Case 2). Several other members of the family were subsequently persuaded to have electrocardiograms taken but none of them would consent to radiological examination. None of the electrocardiograms showed any abnormality.

All three patients had gross cardiomegaly (unfortunately an X-ray examination was not performed on Case 3), and the electrocardiograms showed some form of heart block, greatly increased amplitude of complexes and changes in the $\mathrm{S}-\mathrm{T}$ segments and $\mathrm{T}$ waves suggesting considerable muscular hypertrophy and probable ischæmia. In view of the findings in Evans' series, Case 1 had a biopsy of the vastus externus muscle and this showed heavy glycogen deposits. He died in a Stokes-Adams attack on January 8, 1953, and a post-mortem examination was carried out.

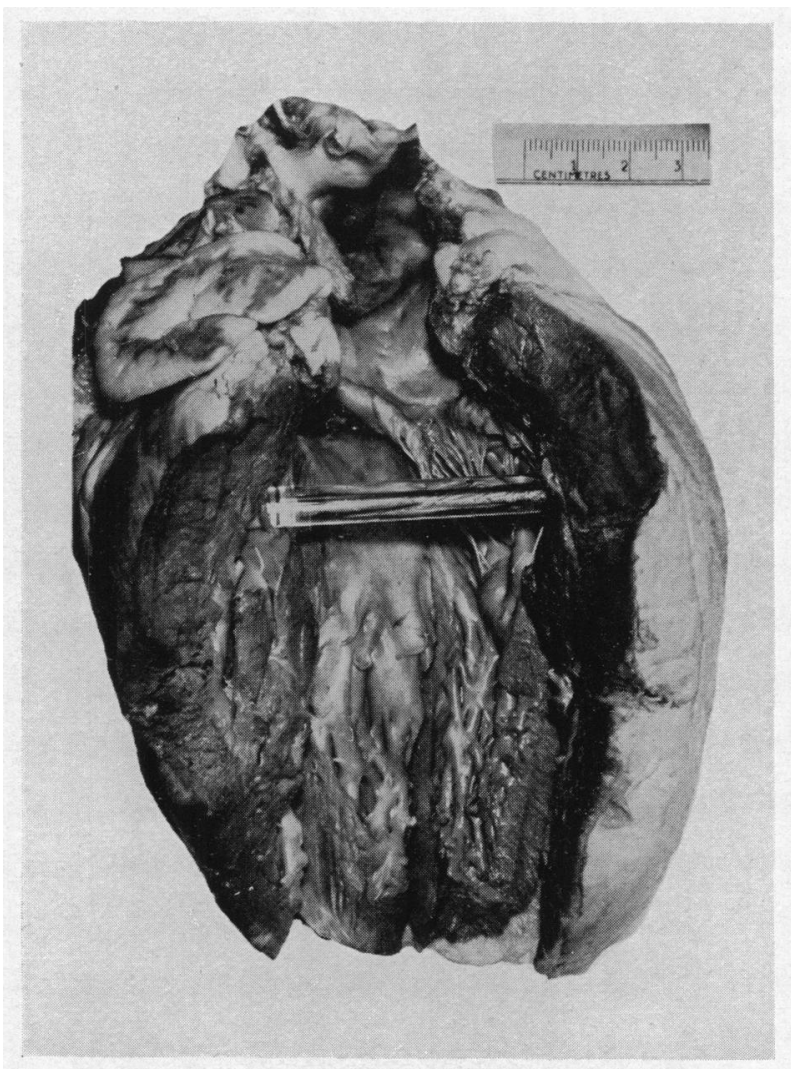

FIG. 4.-Heart of Case 1 weighing $737 \mathrm{~g}$. There is general hypertrophy, especially of the left ventricle.

Case 3 refused further investigation and subsequently died at home; permission for post-mortem examination could not be obtained. Case 2 remained well except for slight dyspnœa on severe exertion; she has since married and left the district.

Necropsy Report on Case 1. Externally, the subject was moderately well nourished and middleaged; there was no abnormality of note. Internally, the lungs presented a moderate degree of emphysema but otherwise they appeared normal. The heart was much enlarged (Fig. 4) and weighed 737 grams. The walls of all the chambers were hypertrophied, that of the left ventricle being up to $22 \mathrm{~mm}$. in thickness. This measurement is of the wall proper and excludes the papillary muscles, which were also very much hypertrophied. The myocardium presented no 
macroscopic abnormality. The valves were normal, as were the septa and the auricular appendages. The coronary arteries presented only a mild degree of atheroma. Nothing else of note was discovered in the heart. The aorta showed a little atheroma. The gall-bladder presented a moderate degree of cholesterolosis and its bile contained a little biliary gravel. All other organs appeared normal macroscopically.

Microscopically, the appearances of the myocardium constitute the main interest of the case. In the sections stained by hæmatoxylin and eosin the most striking feature consists in the presence of multiple vacuoles in the myocardial fibres (Fig. 5). These vacuoles are very prominent in the fibres of the atria, but less so in those of the ventricles. Closer examination reveals well-marked hypertrophy of the individual muscle fibres and this appears to be particularly striking in those fibres showing marked vacuolation.

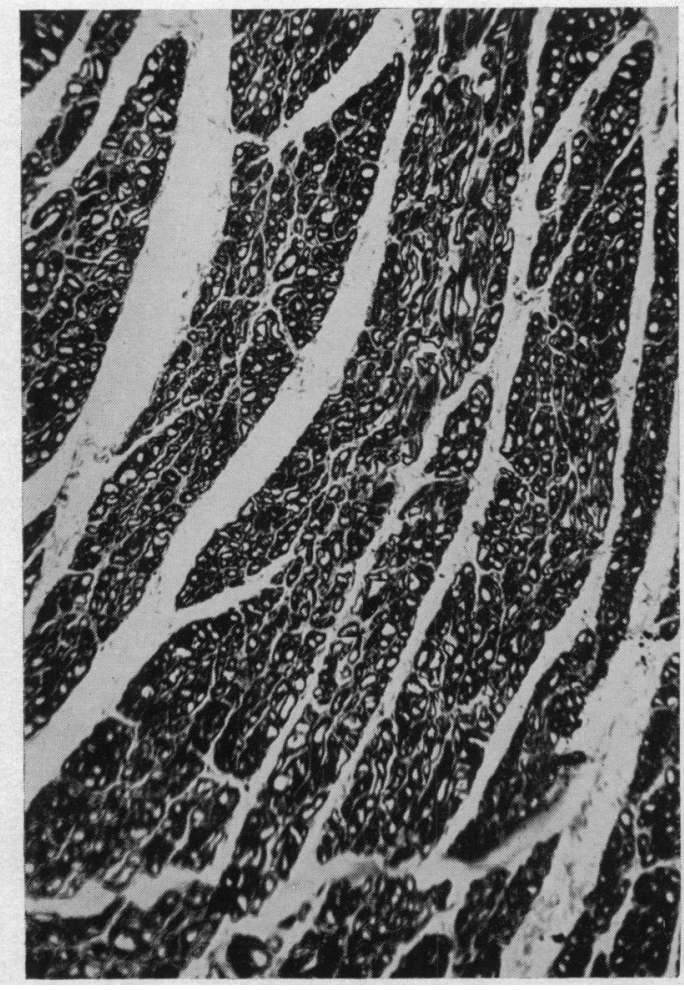

Fig. 5.-Photomicrograph $(\times 80)$ of myocardium of Case 1, showing marked vacuolation of myocardial fibres. H. and $\mathrm{E}$.

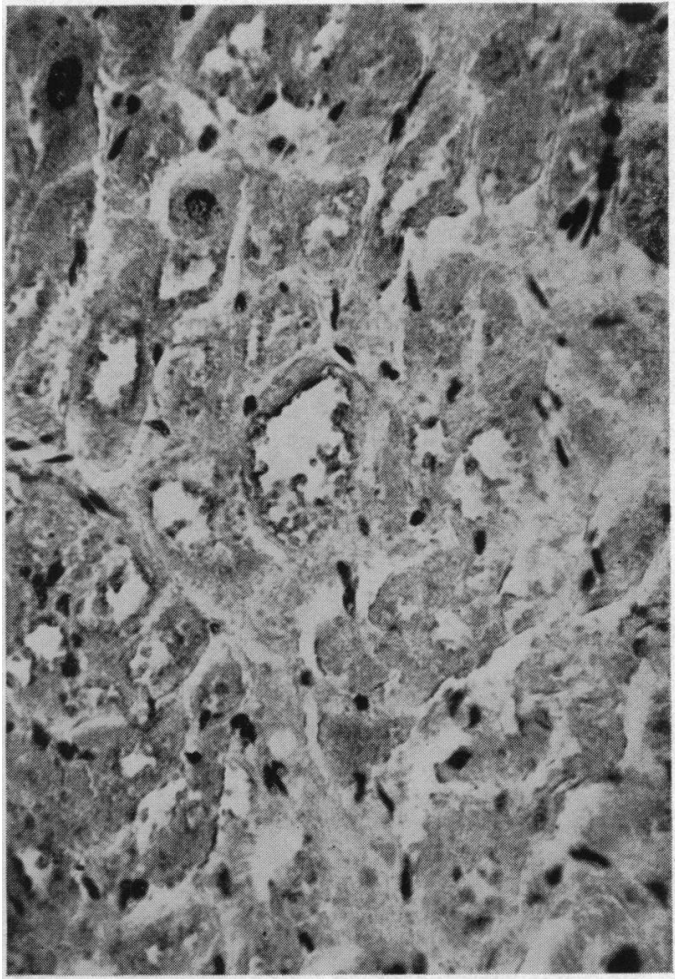

FIG. 6.-Photomicrograph $(\times 360)$ of myocardium of Case 1, showing deposits of glycogen, particularly in the vacuoles.' Best's carmine.

In addition to the hypertrophy and vacuolation of the muscle fibres, there is a moderate degree of patchy fibrosis of the myocardium. In suitably stained sections, traces of fat only were found in the myocardial fibres and none in the vacuoles.

Sections from material fixed in Bouin's solution which preserves glycogen, show conspicuous deposits of this substance in the myocardial fibres (Fig. 6). These glycogen deposits are most plentiful in the vacuoles but they are also evident in other parts of the fibres and in the interstitial tissue. In view of the suggestion put forward by Paulley et al. (1954), H. and E. sections and also sections stained by P.A.S. were searched for the parasite of toxoplasma, but without success. 
Sections of the spleen, kidney, one adrenal gland, pancreas, thyroid gland and brain were all examined for glycogen deposits with negative results. Glycogen deposits were, however, found in the liver, but not more plentifully than in the liver of a control case. A little glycogen was also found in the tongue. Hæmatoxylin and eosin sections of all the tissues mentioned showed a little congestion or nothing of note.

Control specimens were examined of those tissues that showed the presence of glycogen, viz. the myocardium, the muscle of the thigh and the liver. As already mentioned, the same amount of glycogen was found in the liver of the control case as in the case under discussion. The myocardium and peripheral muscle of the control case, however, showed traces of glycogen only. The occurrence of deposits of glycogen in the normal heart and peripheral muscle has been controlled more fully by Russell (1948) who examined at necropsy eleven cases which showed only scanty deposits of glycogen.

Other Pathological Investigations. A biopsy of the vastus externus muscle taken shortly before death also shows a heavy content of glycogen granules in the muscle fibres. No vacuoles were demonstrated. Glucose tolerance tests were carried out on Cases 2 and 3. These were completely normal with a final normal adrenaline response in each case. The alkali reserve was 54.8 vols. $\mathrm{CO}_{2}$ in Case 2, and 61.4 vols. $\mathrm{CO}_{2}$ per $100 \mathrm{ml}$. in Case 3 . The blood cholesterol was $193 \mathrm{mg}$. in Case 2 and $230 \mathrm{mg}$. per $100 \mathrm{ml}$. in Case 3.

\section{DisCUSSION}

This series of cases presents features almost identical with those of Evans' (1949) series. Clinically, symptoms have been extremely slight or non-existent until the terminal stages. The first patient worked as a miner without symptoms until two and a half years before his death. The second patient had no cardiac symptoms and continued symptomless until lost trace of. The third patient began having symptoms three years before her death. Both patients who died had StokesAdams attacks. All three had gross cardiac enlargement without hypertension or valvular disease.

The electrocardiograms showed a great similarity to each other and also to those of the first two cases described by Evans. The main changes are widening of the QRS deflections, alteration in the S-T segments and $T$ waves and the high potential of the complexes. This finding of high potential which is of the order of 2.5 millivolts, is most striking and would appear to be a particular feature of the familial cases. The high potential is probably related to the marked hypertrophy of muscle fibres and possibly to the high glycogen content. The great mass of muscle would doubtless explain the widening of the QRS deflections.

Pathologically the basic abnormality consists in large deposits of glycogen both in the heart muscle and in peripheral muscle. In the case of the heart, hypertrophy and vacuolation of the fibres, together with fibrosis, are also evident. These changes are entirely absent in a control case. They are essentially the same as those in the main case described by Evans (1949), although in his instance fibrosis appeared to be more prominent while vacuolation and glycogen deposits were less prominent than in the present patient.

All these findings being essentially the same as those described by Evans (1949), the cases here presented support his conclusion that this is a distinct and specific syndrome, for which he proposed the name " familial cardiomegaly."

Davies (1952) described a series of cases with familial cardiac disease. Although there are conduction defects in his cases, the electrocardiograms do not show the gross abnormalities which are present in Evans' series, and also in the present series, namely, great increase in amplitude, widening of the QRS deflections and changes in the S-T segments. Furthermore the appearances at necropsy described by Davies are inadequate. The diagnosis in his series therefore does not appear to be fully substantiated from the electrocardiographic or pathological points of view. 
The nature of the condition is somewhat obscure. It is, however, familial and hereditary. There are three conditions with which comparison must be made. The first of these is glycogenic or Von-Gierke's disease, where in a few cases glycogen may be found in the heart, in addition to the liver and kidneys. In Von-Gierke's disease, however, there is a failure in glycogenolysis, as a result of which there is hypoglycæmia, ketosis, and absence of the normal rise in blood sugar after adrenalin, and an abnormal blood sugar curve, this being a little raised and prolonged. These tests were performed on the sister and niece of the patient who came to necropsy, although unfortunately not on the patient himself. All results in both the sister and niece were normal, indicating the absence of any abnormality in glycogenolysis.

The second of the conditions for comparison is Friedreich's ataxia, in which fibrosis and hypretrophy of the surviving fibres is frequently evident in the heart muscle (Evans, 1949; Manning, 1950). No glycogen, however, has so far been discovered in any heart from cases of Friedreich's disease. As fibrosis and hypertrophy of the muscle fibres in the myocardium are in themselves non-specific, it might be considered that there is no real basis for comparison. However, in view of the familial and hereditary nature of both Friedreich's ataxia and the condition under discussion it is possible that they are allied and that future observations will reveal the presence of glycogen in the heart muscle of at least some cases of Friedreich's disease. Roth (1948) quoted by Manning (1950) reports a family in which Friedreich's ataxia affected some members and heart disease (of a familial nature) affected other members.

The third of the similar conditions is progressive muscular dystrophy (myopathy) in which the heart may be involved and show an increase of connective and fatty tissue, with atrophy or hypertrophy of myocardial fibres and also degenerative changes and vacuole formation in these fibres (Storstein and Austarheim, 1955). The authors do not mention whether or not glycogen was present in the vacuoles. The similarity between familial cardiomegaly and progressive muscular dystrophy (also frequently familial or hereditary) when this affects the heart, is thus as noteworthy as that between the former and Friedreich's disease. It would appear therefore that, like Friedreich's disease and the myopathies, familial cardiomegaly is basically a genetically determined condition. It should be clearly understood that in the cases here described there was no clinical suggestion whatsoever that the patients were suffering from any form of myopathy.

Finally, reference must be made to the recent contention by Paulley et al. (1954), that familial cardiomegaly inter alia is probably a manifestation of myocardial toxoplasmosis. There are several objections to this contention. In the first place no toxoplasmas were found in material from the present case, although searched for in suitably stained sections as indicated above. According to Valentine et al. (1953), the parasites are easy to find, being readily visible in $\mathrm{H}$. and $\mathrm{E}$. sections. Secondly, the suggestion is based on inadequate consideration of the histological changes that occur. Thus in the case here described the vacuolation of fibres and the glycogen deposits (not referred to by Paulley et al.) must have a close relationship to the ætiology of the condition and there is no information to suggest that similar or related changes have ever occurred in toxoplasmosis. These abnormalities appear to be specific for familial cardiomegaly.

Thirdly, the suggestion of toxoplasmic ætiology can only be correlated with the familial nature of the condition if it is assumed that a special strain of toxoplasma with myocardial affinity is concerned. This is a suggestion put forward by Paulley et al., but it is speculative and is in disagreement with the protean manifestations of all established cases of toxoplasmosis to which Paulley et al. refer.

Fourthly, the suggestion is based on serological evidence. While the figures given by Paulley et al. for the dye test and complement fixation test in his cases cannot be ignored, they are all low except for the dye test in the third case. The low figures obtained must be balanced against the fact that a low titre of antibodies is found in 28 per cent of the adult population (Valentine et al., 1953). Moreover, recent work has demonstrated the lack of specificity of the dye test in the diagnosis of toxoplasmosis (Awad, 1954). 


\section{SUMMARY}

Three cases of familial cardiomegaly are described, all in members of one family in whom there was gross cardiac enlargement in the absence of any of the usual causes. All three patients showed a remarkable similarity both clinically and electrocardiographically to those previously described by Evans.

Two of the cases had very little cardiac disability prior to the terminal stages of the disease. The third has suffered very little cardiac disability so far.

In the only necropsy performed large deposits of glycogen were found both in the heart and in peripheral muscle and in addition the heart muscle showed hypertrophy, vacuolation of the fibres, and areas of fibrosis.

The condition is discussed and support is given for the view that the cases presented were suffering from familial cardiomegaly. There is some evidence that this condition is allied to Friedreich's ataxia and progressive muscular dystrophy, both the latter and familial cardiomegaly being genetically determined. There is little to support the suggestion that the condition is due to toxoplasmosis.

Grateful acknowledgment is made to Dr. J. L. Grout for the radiological examinations.

\section{REFERENCES}

Addarii, F., Mahaim, L., and Winston, M. (1946). Cardiologia, 11, 36.

Awad, F. I. (1954). Lancet, 2, 1055.

Case Records Massachusetts General Hospital (1942). New England J. Med., 226, 158.

Davies, L. G. (1952). Brit. Heart J., 14, 206.

Evans, W. (1949). British Heart J., 11, 68.

Manning, G. W. (1950). Amer. Heart J., 39, 799.

Paulley, J. W., Jones, R., Green, W. P. D., and Kane, E. P. (1954). Lancet, 2, 624.

Roth, M. (1948). Brain, 71, 416.

Russell, D. (1948), quoted by Evans, W. (1949). Brit. Heart J., 11, 68.

Storstein, O., and Austarheim, K. (1955). Acta med. Scand., 150, 431.

Valentine, J. C., Lane, W. F., Beattie, C.P., and Beverley, J. K. A. (1953). J. Clin. Path., 6, 253. 